\title{
Cardiovascular Changes Induced by Acute Emotional Stress Estimated From the Pulse Transit Time Difference
}

\author{
Pablo Armañac ${ }^{1,2}$, Spyridon Kontaxis ${ }^{1,2}$, Jesús Lázaro ${ }^{3,1,2}$, Pablo Laguna ${ }^{1,2}$, \\ Raquel Bailón ${ }^{1,2}$, Eduardo Gil ${ }^{1,2}$ \\ ${ }^{1}$ BSICoS group, Instituto de Investigación en Ingeniería de Aragón, University of Zaragoza, Spain \\ ${ }^{2}$ Centro de Investigación Biomédica en Red (CIBER), Madrid, Spain \\ ${ }^{3}$ Department of Biomedical Engineering, University of Connecticut, Storrs, CT, USA
}

\begin{abstract}
In the present work, the difference between arrival times of photoplethysmographic (PPG) pulses from two different sites of the arterial tree (PTTD) is calculated. As the PTTD is considered to be related with arterial pulse wave velocity, an evaluation of it's usefulness to identify states of acute mental stress is performed. A subset of fourteen healthy volunteers undergoing a stress protocol was analysed and a statistical test was carried out to evaluate the validity of the proposed method. The results showed that the standard deviation of the PTTD had sufficient statistical capacity to discern between states of stress and relaxation. Furthermore, a generalised descending trend of the mean PTTD can be seen from relaxation to stress.
\end{abstract}

\section{Introduction}

Stress is a physiological process that works as a vital warning system. It boosts memory and helps to perform tasks more efficiently. However, a prolonged stress weakens the immune system and may cause hypertension, fatigue, depression and general deterioration of the heart function. The Autonomic Nervous System (ANS) plays an important role in the regulation between the "rest-anddigest" and the "stress-warning" mode. Under the presence of a stressful stimulus, the body thinks it is under attack and the ANS switches to the "fight or flight" mode, releasing a complex mix of hormones and chemicals (epinephrine, nor-epinephrine and cortisol) in order to shut down unnecessary bodily functions such as digestion, salivation, sexual arousal or urination by the regulation of the blood flow via vasoconstriction and vasodilation. Therefore, by studying the vascular changes produced by this imbalance, a measure of the body's response to stress can be obtained.

One of the parameters than can be used to quantify vascular changes is the pulse transit time (PTT), which is the time that the arterial pressure wave takes to travel along two points of the arterial tree [1]. The non-invasive measurement of PTT is not easy, so pulse arrival time (PAT) is usually used as surrogate. PAT is measured as the time between the heart beat in the electrocardiogram (ECG) to the pulse occurrence in a PPG signal. Methods exploiting features derived from the PAT have been used to provide continuous cardiovascular information [1], and non-invasive blood pressure estimation [2].

However, the use of PAT as PTT surrogate is limited since it includes the pre-ejection period (PEP) [3]. The PEP is defined as the time between the ventricular depolarization and the opening of the aortic valve, that varies beat-to-beat and uncorrelates the PAT-based blood pressure estimation in the current techniques [4]. An alternative PTT surrogate which is independent of the PEP is the PTT difference (PTTD). It can be estimated as the difference between two PATs, or as the time between the pulse occurrence in a PPG signal and the pulse occurrence in a second PPG signal recorded in another place of the body [5]. Actually, the PTTD has already been reported in the literature as a measure correlated with the PTT $[3,5]$. In addition, results in [3] showed higher correlation between PTTD and PAT during rest stages than during tilt stage, when the PEP dynamics have stronger effect in PAT dynamics. In this work, changes in PTTD during accute mental stress are studied in order to assess the potential of PTTD as stress detector.

\section{Methodology}

\subsection{Database}

The database consists of ECG and PPG signals of 14 healthy volunteers ( $22 \pm 3$ years old $)$, not previously diagnosed with any chronic or psychological disease. The $A B P$ 10 module (Medicom 83 system, MTD Ltd, Russia) was used for the simultaneous and synchronized acquisition of the PPG at both the forehead and the finger sampled at 250 $\mathrm{Hz}$, the ECG by 3 orthogonal leads sampled at $1 \mathrm{KHz}$ and respiration effort at $250 \mathrm{~Hz}$. 
Participants underwent a stress-induced session, which lasted 35 minutes, that consisted in a modification of the Trier Social Stress Test (TSST) with an additional arithmetic task [6]. The session included the following stages: 1) $B L_{\mathrm{S}}$, Baseline Relaxing stage. 2) $S T$, Story-Telling stage. 3) $M T$, Memory Test. 4) $S A$, Stress Anticipation. 5) $V E$, Video Exhibition. 6) $A T$, Arithmetic Task. The first and last seconds of each phase were removed to avoid transient phenomena.

\subsection{Preprocessing}

First, PPG signals were band-pass filtered between 0.3 and $15 \mathrm{~Hz}$ with a 4th order Chebyshev type II filter, in order to eliminate the baseline contamination and high frequency noise. Forward-backward filtering was applied for preserving signal morphology.

Then, since the acquisition of the PPG is very sensitive to motion artefacts, an artefact detector was implemented. First, a PPG artefact detector based on the energy of the signal was applied in order to detect motion artefacts that are characterized by higher energy than clean PPG signal segments (see Fig. 1 (top)). Then, remaining artefactual PPG segments were discarded by using an artefact detector based on Hjorth parameters [7].

The energy-based artefact detection is performed in the following steps: First, the PPG signal is squared to emphasize the segments with artefacts related to high-energy. Then, the moving variance, $s(n)$, of the squared PPG is calculated in a window of 2 seconds-length, while the moving median $m(n)$ in a window of 100 seconds-length. When $s(n)>5 * m(n)$, that sample " $n$ " is considered as artefact since a large deviation of the median PPG value occurred.

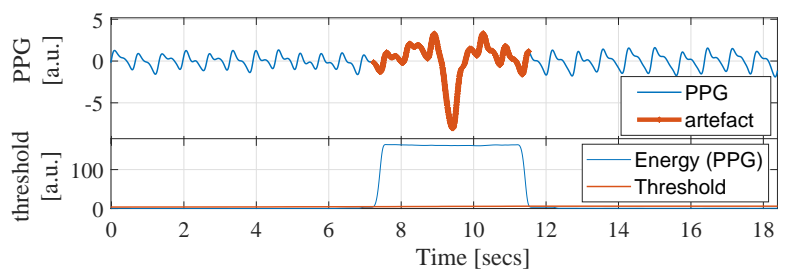

Figure 1. Artefact Detection based on the energy of the signal. PPG signal where artefactual segments were defined at top and Decision criteria at the bottom.

\subsection{Pulse detection}

The time of the maximum of the R-wave for each QRS complex $\left(n_{\mathrm{R}_{i}}\right)$ was detected by using a wavelet-based algorithm [8]. Each PPG pulse was detected by an algorithm that determines the maximum up-slope point of each PPG pulse based on a low pass differentiator filter and a time-varying threshold [9]. The detection of the maximum up-slope instant for the PPG pulses at the forehead and at finger were denoted as $n_{\mathrm{UH}}$ and $n_{\mathrm{UF}}$, respectively, representing the time instant when the PPG pulse have reached the maximum flow velocity during the ventricular systole.

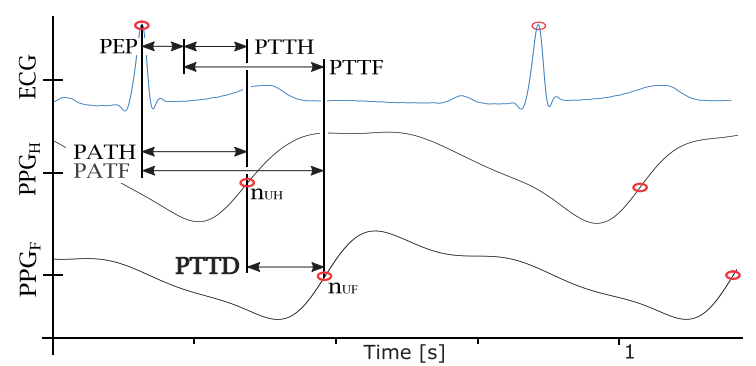

Figure 2. Example of $E C G$, forehead $\left(P P G_{H}\right)$ and finger $\left(P P G_{F}\right)$ PPG signals with the definitions of PATH (Head), PATF (Finger) and PTTD. The intervals of PEP, PTTH (Head) and PTTF (Finger) are unknown but are illustrated for exemplification purposes.

After the pulse detection, the algorithms developed by Mateo et al. [10], to replace ectopic and erroneous beat detections by "normal" beats, were used for the ECG detections and adapted for the detections of the PPG pulses at forehead and at finger.

\subsection{PAT and PTTD estimation}

PAT towards the finger (PATF) was measured as the difference of those fiducial points detected at the finger-PPG signal and those detected in the ECG signal:

$$
x_{\mathrm{PATF}}^{u}(n)=\sum_{i}\left[n_{\mathrm{UF}_{i}}-n_{\mathrm{R}_{i}}\right] \cdot \delta\left(n-n_{\mathrm{UF}_{i}}\right),
$$

where $\delta(\cdot)$ denotes the Kronecker delta function, and the superscript "u" denotes that the signals are unevenly sampled, as the heartbeats occur unevenly in time. The PAT towards the forehead (PATH), $x_{\mathrm{PATH}}^{u}(n)$, was computed similarly, this time using the forehead PPG signal.

On the other hand, the PTTD can be measured as the pulse instant difference:

$$
x_{\mathrm{PTTD}}^{u}(n)=\sum_{i}\left[n_{\mathrm{UF}_{i}}-n_{\mathrm{UH}_{i}}\right] \cdot \delta\left(n-n_{\mathrm{UF}_{i}}\right) .
$$

Some considerations for the equation (2) are:

- A value of $x_{\mathrm{PTTD}}^{u}(n)$ is considered out of the physiological range and excluded from the analysis if it is out of $[-50,150] \mathrm{ms}$ bounds.

- The instants of detections at finger, i.e., the $n_{\mathrm{UF}}$, are considered as the time reference for each $x_{\text {PTTD }}^{u}$ pulse because of its reliability and common use rather than $n_{\mathrm{UH}}$.

- $x_{\mathrm{PTTD}}^{u}(n)$ can be negative, i.e., the pulse gets finger sooner than forehead.

The spurious pulses of the PAT and PTTD estimates were eliminated by means of an outlier detector with the parameters adjusted empirically [11], $N_{e}=10$ and $C=$ 2.75. Subsequently, the evenly-sampled versions were obtained by cubic spline interpolation at $4 \mathrm{~Hz}$ (see Fig. 3), being denoted as $x_{\mathrm{PATH}}(n), x_{\mathrm{PATF}}(n)$, and $x_{\mathrm{PTTD}}(n)$. 


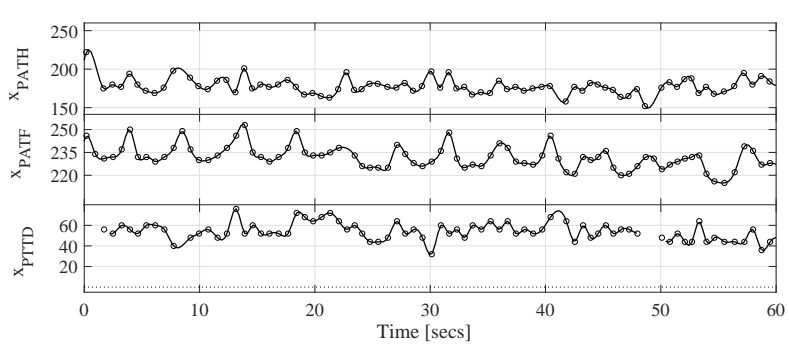

Figure 3. Example of PATH on top, PATF in the medium, and PTTD at bottom. Continuous lines represent the evenly sampled versions. Units in [msecs].

\subsection{Spectral estimation}

A 300-order-FIR band-pass filter was applied to $x_{\mathrm{PTTD}}(n), x_{\mathrm{PATH}}(n)$ and $x_{\mathrm{PATF}}(n)$ series. The cut-off frequencies correspond to the classical bands for heart rate variability (HRV) analysis, as physiological information is expected in PTT in the low frequency $([0.04-0.15] \mathrm{Hz}$, following the Mayer waves [12]), and in the high frequency bands ([0.15 - 0.4] Hz, expected to follow respiration [13]). The resulting signals were analysed in a 30-seconds-length running window with a 50\%-overlap for spectral analysis.

\subsection{Statistical analysis}

The features used are the median and standard deviation, $\sigma$, calculated with a 1 -minute-length sliding window, in order to avoid biased estimation, for each pulse " $i$ " of the $x_{\mathrm{PTTD}}(n), x_{\mathrm{PATH}}(n)$ and $x_{\mathrm{PATF}}(n)$ series. The stages are characterized by the median of the values obtained in each windowed signal. The Wilcoxon signed-rank test (subjectpaired) was performed to study the differences between relax and stress stages. An statistical analysis was applied for finding differences in PTTD-based features between the stress $(S T, V E, S A)$ and relax $\left(B L_{\mathrm{S}}\right)$ stages of the protocol. In addition, the same features were calculated for the PATH and PATF signals in order to compare the power of discrimination.

\section{Results}

The last five stages of the stress session are considered stressful and the psychometric evaluation at the end of the session revealed that subjects responded to the stress stimuli [6]. $M T$ and $A T$ phases were excluded from the analysis because subjects were requested to speak during those phases; as PTT has a strong relation with respiration, changes with respect to basal can be reflecting speakinginduced changes in respiration.

The subset used was extracted from a larger database, where only fourteen subjects had both PPG signals with sufficient quality to be able to calculate the corresponding PTTD with a detection percentage greater than $70 \%$ with respect to the total number of heartbeats. Surprisingly, there were subjects who had a negative basal PTTD,
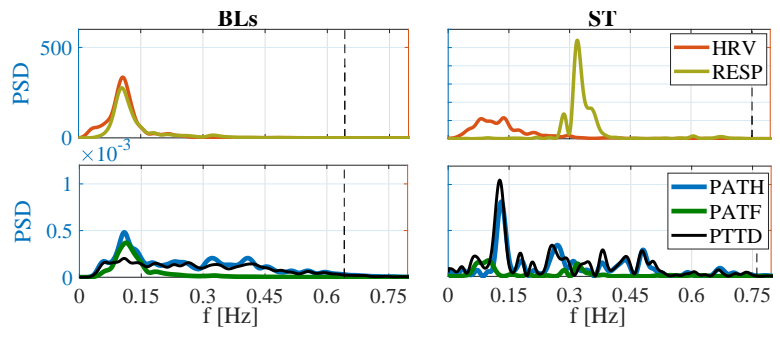

Figure 4. PTTD spectral analysis for a subject in $B L_{\mathrm{s}}$ and $S T$ stages. HRV (from the ECG) with Respiration (top) and PTTD, PATF and PATH spectral estimation (bottom).

which means that the pulse wave was reaching the finger before the forehead in relaxation.

Table 1 shows the median and interquartile range (IQR) obtained by comparing the median and $\sigma$ of the PATH, PATF and PTTD for all the subject among basal stage and the different stress stages of the protocol. Spectral analysis was also performed in order to compute the frequency indices. Although it could only be done for the whole subset since the duration of the evenly-sampled signals without time gaps were too short to be done, one example is presented in Figure 4.

\section{Discussion}

The possibility of quantifying changes in the sympathovagal balance caused by acute mental stress was studied, using non-invasive sensors based on PPG and also without the need of either the ECG or cuff/intra-arterial BP sensors in the case of the PTTD index. As shown in Table 1, statistically significant differences for the variance of PTTD were found in the $S T$ when compared to $B L_{\mathrm{s}}$, suggesting that it might be useful to detect states of acute mental stress.

Although there were no statistically significant differences in the median -that could be due to the small number of subjects-, a descending trend may be observed in the stressful stage $S T$ compared to the relax stage $B L_{\mathrm{s}}$ suggesting that the PTTD decreases with stress. Furthermore, statistically significant reduction in the median of both PATs was seen in $S T$ and $V E$ with respect to $B L_{\mathrm{s}}$. According to the results in [6], $S T$ and $V E$ were also more differentiable than $S A$ because of the specific stressors. With this mechanism, the pulse wave velocity to the limbs is greatly increased with respect to the one to the forehead despite the fact that the volume of blood that reaches to the brain is much greater than to the hands.

Spectral analysis shown in Fig. 4 reveals that the PATF (blue) has less power than the PATH (green) during stressful stimuli $(S T)$ and, therefore, the power of PTTD (orange) is very similar to that of the PATH. On the contrary, PAT spectral power in relaxation $\left(B L_{\mathrm{S}}\right)$ is similar for both PATF and PATH, resulting in a lower PTTD power. Moreover, in the basal stage, it is clearly seen that there is a component around the respiratory frequency in both PATs and PTTD, related to Respiratory Sinus Arrhythmia. However, 


\begin{tabular}{c||c|ccc} 
Parameters [msecs] & $B L_{\mathrm{S}}$ & $S T$ & $S A$ & $V E$ \\
\hline \hline median (PTTD) & $26(16-36)$ & $18(4-34)$ & $24(12-40)$ & $21(0-40)$ \\
median (PATH) & $211(200-245)$ & $\mathbf{1 9 2}(\mathbf{1 6 2 - 2 2 3})^{*}$ & $208(185-245)$ & $\mathbf{2 0 3}(\mathbf{1 7 5 - 2 3 3})^{*}$ \\
median (PATF) & $248.5(235-262)$ & $\mathbf{2 2 2}(\mathbf{2 1 1 . 5 - 2 2 7})^{*}$ & $247(229-261)$ & $\mathbf{2 3 5}(\mathbf{2 2 1 - 2 5 3})^{*}$ \\
\hline$\sigma($ PTTD) & $9.84(6.7-14)$ & $\mathbf{1 2}(\mathbf{1 0 . 5 - 1 7 . 7 0})^{*}$ & $14(7.5-15)$ & $11(9.6-11.6)$ \\
$\sigma$ (PATH) & $11(9-20)$ & $11(8-15)$ & $13(9-18)$ & $10(9-19)$ \\
$\sigma$ (PATF) & $6.3(5.6-7.6)$ & $6.2(4-7.1)$ & $6.6(6.3-8)$ & $5.5(5-6.7)$ \\
\hline \hline
\end{tabular}

Table 1. Median $\left(I Q R_{25 \%}-I Q R_{75 \%}\right)$ for the moving median and $\sigma$ with a 1 minute-length window for PTTD, PATH, PATF in relax and stressful stages. Statistical significance between a stressful stage and $B L_{\mathrm{s}}$ is marked with an asterisk.

in the stress stage, the PATF has two components, one below $0.15 \mathrm{~Hz}$ and another around the respiratory frequency; while almost all the power of PATH is centred around 0.1 $\mathrm{Hz}$, apparently associated with the Mayer wave which is a resonant effect of vasoconstriction with a period of $10 \mathrm{sec}-$ onds caused by acute sympathetic activations [12]. This Mayer wave, being very powerful at forehead but not at finger, are reflected in the PTTD as a consequence. A similar finding was obtained in [3] for 14 subjects that performed a tilt-table test. During tilt stage, the power in the LF band at forehead was substantially increased with respect to the one at finger, thus in the LF power of the PTTD.

One possible hypothesis to explain that increase of the Mayer wave power in stress stages only in PATH, hence in PTTD, is the following: During a stressful situation, sympathetic activation causes vasoconstriction in the blood vessels, whose effect is expected to be much greater in the extremities than in the vessels that carry blood to the brain. Then, probably, the rigidity of the peripheral arteries of the extremities under acute sympathetic activations is much greater, thus a lower dynamic range of PATF relative to that of PATH might be expected during stress. On the contrary, during relax there is an absence of such strong vasoconstriction and the variations in the PAT that can be observed in the finger and in the forehead are quite similar.

Nevertheless, it's necessary to verify if the existence of all these components is consistent and generalizable. A study involving more subjects is required as the PPG signals registered at forehead in this database were of low quality. Then, only few of them had a reliable spectral estimation from a PTTD signal without time gaps due to the large amount of bad or miss-detections.

This methodology could be embedded in ad-hoc devices for the PTTD measurement. It may lead to clinical applications that include not only stress assessment and identification but also non-invasive, continuous and ambulatory monitoring of the arterial tree condition with the need of only two PPG sensors. However, the noise sensitivity of the PPG is a significant aspect to take into account.

\section{Conclusions}

It can be concluded that the standard deviation of the PTTD is able to distinguish between relax and induced mental stress. Local vasoconstrictions caused by Mayer waves could explain the significant strong fluctuations in the PTTD. In addition, a descending trend is also observed in the PTTD in the case of a sympathetic activation.

\section{Acknowledgements}

This project has received funding from the EU Programme H2020 under the Marie Sklodowska-Curie Grant No.745755; by Gobierno de Aragón (Reference Group BSICoS T39-17R) cofunded by FEDER 2014-2020; by project RTI2018-097723-B-I00 and by CIBER-BBN.

\section{References}

[1] Smith R, et al. Pulse Transit Time: an appraisal of potential clinical applications. Thorax 1999; 54(5).

[2] Ding XR, et al. Continuous cuffless blood pressure estimation using pulse transit time and photoplethysmogram intensity ratio. IEEE TBME 2016; 63(5).

[3] Lázaro J, et al. Difference in pulse arrival time at forehead and at finger as surrogate of pulse transit time. CinC. 2016.

[4] Newlin DB. Relationships of pulse transmission times to pre-ejection period and blood pressure. Psychophysiology 1981; 18(3).

[5] Nitzan M, et al. The difference in pulse transit time to the toe and finger measured by photoplethysmography. Physiological measurement 2001; 23(1).

[6] Hernando A, et al. Inclusion of respiratory frequency information in heart rate variability analysis for stress assessment. IEEE JBHI 2016; 20(4).

[7] Peralta E, et al. Optimal fiducial points for pulse rate variability analysis from forehead and finger photoplethysmographic signals. Physiological measurement 2019; 40(2).

[8] Martínez JP, et al. A wavelet-based ECG delineator: evaluation on standard databases. IEEE TBME 2004; 51(4).

[9] Lázaro J, et al. Pulse rate variability analysis for discrimination of sleep-apnea-related decreases in the amplitude fluctuations of pulse photoplethysmographic signal in children. IEEE JBHI 2014; 18(1).

[10] Mateo J, Laguna P. Analysis of heart rate variability in the presence of ectopic beats using the heart timing signal. IEEE TBME 2003; 50(3).

[11] Bailón R, et al. A robust method for ECG-based estimation of the respiratory frequency during stress testing. IEEE TBME 2006; 53(7).

[12] Julien C. The enigma of Mayer waves: facts and models. Cardiovascular Research 2006; 70(1).

[13] Chua C, Heneghan C. Pulse transit time-derived respiratory parameters and their variability across sleep stages. In IEEE-EMBS. 2006.

Address for correspondence:

Pablo Armañac, (parmanac@unizar.es). c/ María de Luna, Ed. Ada Byron, L.3.07; 50018, Universidad de Zaragoza (Spain). 